\title{
Sistemas homogéneos de ecuaciones diofánticas. Resolución y aplicación al ajuste automático de reacciones químicas
}

\author{
ALFONSO RECUERO, Dr. Ing. Caminos, Canales y Puertos \\ J. PEDRo GUTIERREZ, Ing. Caminos, Canales y Puertos \\ (IETCC/CSIC)
}

\section{$R E S U M E N$}

Se presenta un algoritmo de resolución de sistemas de ecuaciones diofánticas lineales y homogéneas. Seguidamente y como ejemplo de aplicación se describe un método de búsqueda heurística de una solución óptima que cumpla unas ciertas condiciones dentro de un espacio infinito de soluciones de varias dimensiones. Por último se describe la aplicación del algoritmo y del procedimiento de búsqueda heurística para el caso de ajuste de reacciones químicas. Se incluye el listado en BASIC del programa para el ordenador $\mathrm{HP}-75$

\section{$S U M M A R Y$}

An algorithm to solve systems of linear homogeneous diophantine equations is given here. Subsequently, as an example of application, a method of heuristic search for the best solution is discribed; this fulfils certain conditions within an infinite space of solutions of various dimensions. Finally, an application of the algorithm and of the process of heuristic search for the best solution in adjusting chemical reactions is described. The listing in BASIC of the program of application mentioned is included, for the HP-75 portable computer.

\section{IN T R O D U C C I O N}

Se denominan diofánticas a aquellas ecuaciones de coeficientes enteros que admiten infinitas soluciones de las que nos interesan solamente las que corresponden a valores enteros de las variables. Tendremos un sistema de tales ecuaciones cuando deban verificarse simultáneamente varias ecuaciones de este tipo.

Esta clase de sistemas de ecuaciones se presentan, por ejemplo, en aquellos casos en los que se desea obtener un cierto número de conjuntos a partir de otro grupo de conjuntos de forma que todos y cada uno de los componentes de los conjuntos de entrada estén en los de salida. El ejemplo más típico es el ajuste de reacciones quimicas, en las que a partir de unas ciertas moléculas de entrada se obtienen otras de salida de modo que todos los átomos de las moléculas de entrada estén en las de salida.

En el presente trabajo se describe un método general de resolución de sistemas homogéneos de ecuaciones diofánticas, incluyendo un procedimiento heuristico para la búsqueda de una solución óptima cuando el conjunto de soluciones tiene varios grados de libertad, asi como la implementación del mismo en un programa escrito en BASIC para la calculadora HP-75 C.

Como ejemplo de aplicación se utiliza el ya citado de ajuste automático de reacciones quimicas incluyéndose un programa en BASIC que realiza dicho ajuste. 


\section{RESOLUCION DE UN SISTEMA HOMOGENEO DE ECUACIONES DIOFANTICAS}

Se considera el sistema de $E$ ecuaciones con $V$ variables dado por

$$
K X=0
$$

donde $K$ es una matriz de coeficientes enteros, y se desean obtener las soluciones del mismo en las que las componentes de $X$ sean asi mismo enteras.

El sistema debe ser indeterminado, pues en caso contrario solo admitiria la solución trivial $X=0$.

Si denominamos $R$ al rango de la matriz $K$, el conjunto de soluciones del sistema formará un espacio vectorial de $V-R$ dimensiones como máximo. El algoritmo que se describe permite la determinación de una base de este espacio vectorial de soluciones formadas por vectores cuyas componentes son todas números enteros, de modo que el $M C D$ de las componentes de cada solución es 1. Cualquier solución entera del sistema se podrá expresar como una combinación lineal de las soluciones básicas multiplicadas por escalares enteros.

El algoritmo descrito está inspirado en el método de eliminación de Gauss, en el que se han introducido las modificaciones adecuadas con objeto de trabajar siempre con coeficientes enteros.

Se utilizan dos vectores auxiliares de direccionamiento $L$ y $C$, de $E$ y $V$ componentes respectivamente, los cuales son inicialmente puestos a cero.

La primera etapa del proceso es la triangularización. En él se trata de encontrar una ecuación $I$ que sirva de base para la determinación de la variable $X(J)$, de modo que en dicha ecuación intervengan exclusivamente las variables $X(L), L=J, \ldots V$. Para ello se procede ordenadamente, variable por variable buscando entre las ecuaciones que no hayan servido de base para una variable anterior, la primera en la que el coeficiente $K(I, J)$ sea distinto de cero. Una vez encontrada se utiliza esta ecuación para anular todos los coeficientes $K(L, J)$ en aquellas ecuaciones $L$ que no hayan servido de base para una variable anterior. En este caso, se marcarán los vectores de direccionamiento con $L(I)=J$ y $C(J)=I$, indicando así que la ecuación $I$ es la base para determinar la componente $J$, y que la componente $J$ se determina con la ecuación $I$.

En caso de no encontrar ningún coeficiente $K(I, J)$ distinto de cero en el proceso anterior, la variable $J$ se considerará como variable independiente.

El número máximo de variables dependientes será:

$$
N V D=\min (E, V-1)
$$

Cada variable dependiente tendrá una ecuación base asociada. Las restantes variables serán consideradas independientes. El vector $C$ permitirá diferenciar las mismas, ya que $C(J)$ será cero cuando $X(J)$ sea independiente, y distinto de cero en caso contrario.

El proceso de triangularización se sigue hasta que se hayan determinado las ecuaciones base de hasta $N V D$ variables. En caso de que queden ecuaciones que no hayan servido como base de alguna variable, si todos sus coeficientes no fuesen nulos el sistema no sería indeterminado, y en consecuencia admitiría exclusivamente la solicitud trivial.

A continuación se pasará al proceso de sustitución hacia atrás. Se harán también tantos procesos de este tipo como variables independientes contenga el sistema, en cada uno de los cuales se asignará inicialmente el valor 1 a una de las variables independientes y cero a las restantes. 
Se tomará entonces las ecuaciones base de las componentes $X(J) J=V, \ldots 1$ y se determina una solución parcial haciendo:

$$
\begin{gathered}
X(J)=\sum_{\mathrm{L}=\mathrm{J}+1}^{\mathrm{V}} K(I, L) X(L) \\
X(L)=X(L) \cdot K(I, J) \quad L=J+1, \ldots, V
\end{gathered}
$$

y dividiendo posteriormente esta solución parcial por el $M C D$ de las componentes no nulas del vector $X$, desde la $J$ hasta la $V$.

Al final de cada uno de estos procesos de sustitución hacía atrás se obtendrá una solución del sistema que formará parte de la base de posibles soluciones.

\section{BUSQUEDA HEURISTICA DE UNA SOLUCION OPTIMA}

En un cierto número de problemas la solución buscada debe estar formada por enteros mayores que 0 y lo más pequeños posible. Cuando el espacio de solución es unidimensional, el algoritmo descrito proporciona directamente la solución. Sin embargo, cuando el espacio de soluciones es de más de una dimensión, el algoritmo proporciona exclusivamente una base de dicho espacio de soluciones. A continuación se propone un método heuristico, que se muestra eficaz en gran número de ocasiones, para determinar una solución de las caracteristicas citadas a partir de las soluciones básicas proporcionadas por el algoritmo.

Al describir el algoritmo se clasificaron las variables en dependientes e independientes. En cada una de las soluciones básicas una y solo una de las variables independientes es distinta de 0 . Teniendo en cuenta esta peculiaridad de las soluciones básicas generadas, el proceso propuesto es el siguiente:

1) En cada una de las soluciones básicas, detectar el valor de la variable independiente no nula de la misma. Si dicho valor es negativo, cambiar el signo a dicha solución. Determinar el MCD de los valores no nulos de las variables independientes. Sea $L$ dicho MCD.

2) Generar todas las combinaciones lineales de las soluciones básicas así modificadas, multiplicando cada una de ellas por un coeficiente comprendido entre 1 y $L$. En cãda combinación analizada la solución se generará componente a componente, comprobando al obtener cada uno de ellos que es mayor que 0 y que es divisible por $L$. Cuando alguna de las componentes no cumpla este requisito se desechará la combinación y se pasará a la siguiente.

3) La primera solución obtenida que pase por el filtro citado se reducirá, dividiéndola por el MCD de sus componentes el cual será $L$ o un múltiplo de $L$. Esta solución se propondrá como la óptima.

\section{EJEMPLO: AJUSTE DE REACCIONES QUIMICAS}

Un problema cuya resolución conduce al planteamiento de un sistema homogéneo de ecuaciones diofánticas es el de ajuste de reacciones químicas. En este caso se tiene un conjunto de compuestos que reaccionan para dar otro conjunto de compuestos, siendo conocida la composición atómica de todos ellos. El ajuste de la reacción se hace estableciendo que para cada tipo de átomo el balance en ambos grupos sea igual. En el caso particular de reacciones de oxidación reducción es frecuente que alguno de los cumpuestos sean iones, debiendo establecerse en este caso además cl balance de cargas eléctricas.

Se plantea así un sistema de ecuaciones diofánticas, con tantas ecuaciones como tipos de átomos 
intervienen, más uno en el caso de establecer el balance de cargas eléctricas, y con tantas incógnitas como tipos de 'compuestos intervienen.

Veamos algunos casos particulares:

\section{Ejemplo 1}

$$
X \text { (1) } \mathrm{Pt} \mathrm{Cl}_{4} \mathrm{Na}_{2}-X \text { (2) } \mathrm{Pt}+X \text { (3) } \mathrm{PtCl}_{6} \mathrm{Na}_{2}+X \text { (4) } \mathrm{CINa}
$$

\begin{tabular}{|c|c|c|c|c|c|}
\hline \multirow{4}{*}{ BALANCE DE } & & $X(1)$ & $X(2)$ & $X(3)$ & $X(4)$ \\
\hline & $\mathrm{Pt}$ & 1 & -1 & -1 & 0 \\
\hline & $\mathrm{Cl}$ & 4 & 0 & -6 & -1 \\
\hline & $\mathrm{Na}$ & 2 & 0 & -2 & -1 \\
\hline
\end{tabular}

3 ecuaciones con 4 incógnitas

Número soluciones básicas: 1

Solución básica: $X(1)=2 ; X(2)=1 ; X(3)=1 ; X(4)=2$.

\section{Ejemplo 2}

$$
\begin{aligned}
& X(1) \mathrm{Cr}_{2} \mathrm{O}_{7}^{2-}+X(2) \mathrm{Fe}^{2+}+X(3) \mathrm{H}^{1+} \\
& X(4) \mathrm{Cr}^{3+}+X(5) \mathrm{Fe}^{3+}+X(6) \mathrm{H}_{2} \mathrm{O}
\end{aligned}
$$

\begin{tabular}{|c|c|c|c|c|c|c|}
\hline & $X(1)$ & $X(2)$ & $X(3)$ & $X(4)$ & $X(5)$ & $X(6)$ \\
\hline $\mathrm{Cr}$ & 2 & 0 & 0 & -1 & 0 & 0 \\
\hline $\mathrm{O}$ & 7 & 0 & 0 & 0 & 0 & -1 \\
\hline $\mathrm{Fe}$ & 0 & 1 & 0 & 0 & -1 & 0 \\
\hline $\mathrm{H}$ & 0 & 0 & 1 & 0 & 0 & -2 \\
\hline Cargas & -2 & +2 & +1 & -3 & -3 & 0 \\
\hline
\end{tabular}

BALANCE DE

5 ecuaciones 6 incógnitas

Numero de soluciones básicas: 1

Solución básica: $X(1)=1 ; X(2)=6 ; X(3)=14 ; X(4)=2 ; X(5)=6 ; X(6)=7$.

\section{Ejemplo 3}

$$
X(1) \mathrm{Cr}_{2} \mathrm{O}_{7} \mathrm{~K}_{2}+X(2) \mathrm{SO}_{4} \mathrm{H}_{2}+X \text { (3) } \mathrm{SH}_{2}
$$

$$
X(4)\left(\mathrm{SO}_{4}\right)_{3} \mathrm{Cr}_{2}+X(5) \mathrm{SO}_{4} \mathrm{HK}+X(6) \mathrm{S}+X(7) \mathrm{H}_{2} \mathrm{O}
$$

BALANCE DE

\begin{tabular}{l|ccccccc}
\multicolumn{1}{c}{} & $\mathbf{X}(\mathbf{1})$ & $\mathbf{X}(2)$ & $\mathbf{X ~ ( 3 )}$ & $\mathbf{X}(\mathbf{4})$ & $\mathbf{X ~ ( 5 )}$ & $\mathbf{X}(6)$ & $\mathbf{X ~ ( 7 ) ~}$ \\
\cline { 2 - 9 } & $\mathrm{Cr}$ & 0 & 0 & 2 & 0 & 0 & 0 \\
$\mathrm{O}$ & 2 & 4 & 0 & 12 & 4 & 0 & 1 \\
$\mathrm{~K}$ & 7 & 0 & 0 & 0 & 1 & 0 & 0 \\
$\mathrm{~S}$ & 2 & 0 & 1 & 3 & 1 & 1 & 0 \\
$\mathrm{H}$ & 0 & 2 & 2 & 0 & 1 & 0 & 2
\end{tabular}

5 ecuaciones 7 incógnitas.

Número soluciones básicas: 2 


$\left\{\begin{array}{rrrrrrrr}\multicolumn{1}{c}{} & \mathbf{X ~ ( 1 )} & \mathbf{X ~ ( 2 )} & \mathbf{X ~ ( 3 )} & \mathbf{X ~ ( 4 )} & \mathbf{X ~ ( 5 )} & \mathbf{X}(6) & \mathbf{X ~ ( 7 ) ~} \\\right.$\cline { 3 - 9 } 2 & -4 & -13 & 9 & -4 & -8 & 16 & 0 \\ & 4 & 17 & 3 & 4 & 8 & 0 & 16 \\ & 1 & 5 & 3 & 1 & 2 & 3 & 7\end{array}

Los coeficientes de ponderación probados en la búsqueda heurística para las soluciones básicas 1 y 2 , han sido sucesivamente las parejas:

$(1,1),(1,2),(2,2),(1,3),(2,3),(3,3),(1,4),(2,4),(3,4),(4,4),(1,5),(2,5)$, $(3,5),(4,5),(5,5),(1,6),(2,6),(3,6),(4,6),(5,6),(6,6),(1,7),(2,7),(3,7)$,

La pareja $(3,7)$ ha proporcionado la solución propuesta.

\section{PROGRAMACION DEL ALGORITMO}

El algoritmo descrito ha sido programado en BASIC para una calculadora HP-75 C, adjuntándose un listado del programa.

El algoritmo se desarrolla en el programa DIOFAN, que está preparado para ser utilizado como subrutina. Recibe los datos en un archivo llamado DATDIO que contiene, en forma secuencial, el número de ecuaciones, el número de incógnitas, y los coeficientes del sistema ordenados por filas. Devuelve la solución o soluciones básicas en el archivo SOLDIO que contiene, de forma secuencial, el número de soluciones básicas y los componentes de cada una de las soluciones.

Como ejemplo de aplicación se incluye así mismo el programa AJUSTE, el cual pide como datos el número de moléculas de entrada y de salida, y a continuación la fórmula de cada una de las moléculas, que debe darse en la forma tradicional pero sin paréntesis, escribiendo los símbolos de los elementos, con mayúsculas y minúsculas, seguidos del número de átomos si son más de uno. En el caso de iones, las cargas eléctricas del mismo deberán introducirse precedidas de un símbolo de elemento ficticio, $X x$, por ejemplo, seguido del número de cargas, sin signo si son positivas, y precedido del signo - si son negativas.

El programa genera automáticamente la matriz de coeficientes, y presenta la relación de átomos detectados, a efectos de comprobación. A continuación escribe los datos en el archivo DATDIO, pasa el control al programa DIOFAN, y lee los resultados del archivo SOLDIO. Si el número de soluciones básicas es mayor que 1 , realiza la búsqueda heurística de la solución óptima. En caso de que la solución sea única, o que encuentre una solución óptima en la búsqueda, presenta los coeficientes de la solución, y a continuación escribe la ecuación química ya ajustada.

En caso que el sistema no tenga solución lo indicará mediante un mensaje. También indicará con un mensaje el que no haya tenido éxito en la búsqueda heurística de la solución.

\section{REFER E N CI A S}

(1) BAREISS, E. H.: "Computational solutions of matrix problems over an integral domain". J. Inst. Maths. Applics. 10,68-104. 1972.

(2) HILKINSON, J. H.: "Some recent advanced in numerical linear algebra". The state of the art in numerical analysis Edited by D. Jacob. Academic Press 1977, London. 


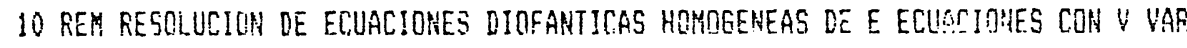
IABLES

20 OPTION EASE 1

30 INTEGEF $X(10, i 0), L(10), C(10), Y(10)$

40 ASSIGN 1 TO "DATDIO"

50 READ $11 ; E, V$

60 FOF $I=1$ TOE

70 FOF $j=1$ TIJ $V$

ED READ \# $1 ; K(1, J)$

90 RE $\mathrm{NET} \mathrm{J}$

100 MEXT I

110 FOR $I=1$ TOE E $($ (I) $=0$ MEXT I

$120 \mathrm{FOR} J=1$ TO $V$ \& $[(\mathrm{J})=0$ \& HEXT $J$

$130 \mathrm{FQB} J=1$ TO $\mathrm{V}$

140 FOR $I=1$ TO $E$

150 IF $L(I)=0$ AHID $K(I, J)$ HO THEV $L(I)=J$ \& $[(d)=I$ E $60 T 0180$

160 NEXT I

170 60T0 290

$180 \mathrm{FOT} I I=1$ TU E

190 IF $L(! 1) \$ 0$ OR $K(I 1, J)=0$ THEN 280

$200 F(1=Y(1, J) \in Y 2=K(11, J)$ \& $M=0$

210 FOR $J 1=\mathrm{J}$ TO $!$

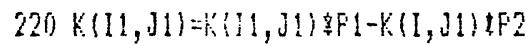

$230 \quad H=F(M(H, k(I I, J 1))$

240 NEXT J1

250 Fon $J 1=j+1$ TO $\mathrm{V}$

260 If $M$ ThEH $R(11,31 i=k(11, j 1) / \mathrm{H}$

270 HEXT II

280 NEYT II

290 NEXT J

$300 \quad S=V$

310 fOR $j=110 \%$

320 If Cold THEN $s=5-1$

330 MEXT J

340 ASSIGN \# 1 TO:

350 ASS16H 1 TO "SOLDIO"

350 FFINT $1 ; 5$

370 FOR $51=1$ TO $\mathrm{s}$

$30 \quad 52=0$

390 FOR $J=1$ TO $V$

100 IF $C(1)=0$ THEN $52=52+1$

410 IF $S ?=51$ THEN $X(J)=1$ ELSE $X(J)=0$

420 NEYT J

430 FOR $J=V$ TH I STEP -1

$440 I=[(\mathrm{J})$

450 IF $]=0$ THEN 590

$\{6052=0$

$470 \quad \mu=0$

$490 \mathrm{FOR} J 1=\mathrm{J}+1$ TO $\mathrm{V}$

$47052=2+k(1, J 1) i) ! J !)$

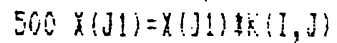

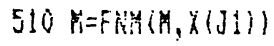

520 NEKT J1

$530 \times(j)=-52$

$540 K_{1}=F N(Y)(Y, X(J))$

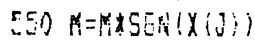

560 fOR $J:=3$ To y

570 IF $M$ THE!: $Y(3)=x(J 1) / M$

580 REXT jI

590 NEXT J 
s00 Fon $j=1$ TO $\mathrm{Y}$

b10 PFIIIT 1 ; X(j)

$\$ 20$ KEXT i

dSU MEXT SI

640 E⿰氵IN

S50 DEF FNM ( $\bar{H}, \mathrm{~B})$

$660 A 1=A B S(A) \& B 1=A E S(B)$

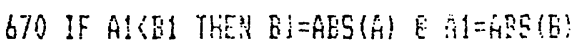

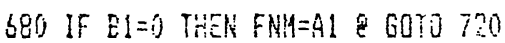

690 Cl= HOL $\left(\mathrm{H}_{1}, \mathrm{~B}_{\mathrm{i}}\right)$

700 If Cl THES $A i=\$ 1$ \& $81=C 1$ e EnTD 690

$710 \mathrm{FH}=\$ 1$

720 END DEF

$10 !=$

20 FEM AUUSTE AUTOMATICO DE UHA ECUACION QUIMICA

30 OFTIOU EASE 1

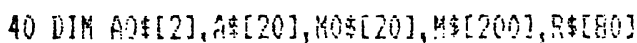

50 l:Terer $x(10,10), x(10), P(10)$

$00 \mathrm{E}=0 \mathrm{U}$ \& $\mathrm{A} F=\mathrm{Pa}$

70 IHFUT "MOLECULAS ENTAADA , SALIDAR",MI, M2

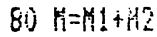

90 For $I=1$ I0 10

loj FOR $J=1$ TO 10

$110 k(I, J)=0$

120 MEXT J

130 HEXT I

140 FOR $J=1$ TO $H$

!50 IF J'M! THEN DISP "MOLECULA SALIDA"; J-H1;ELSE DISP "MOLECULA ENTRADA";

160 INFUT HOS

170 मे $\{[201]-19,20 \div]]=100$

$180 \mathrm{~L}=$ LEN(MOS)

190 FOR $N=1$ TOL

$200 \mathrm{AOS}=H 0+[\mathrm{N}, \mathrm{B}]$

210 IF $H=L$ THEH AOS=AO\$:" "E GOTO 230

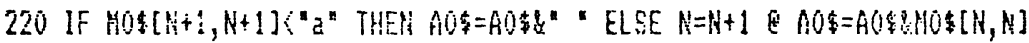

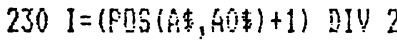

240 IF I $=0$ THEN $E=E+1$ \&I=E $E A=A$ A

250 If $H=L$ IHEN $K(1, J)=1$ e EOTO 320

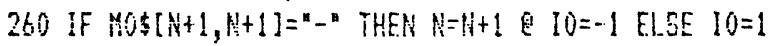

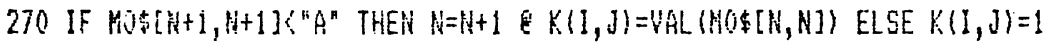

$280 \quad K(1, \pi)=(1,1]) \$$,

270 if $N=L$ THEN 320

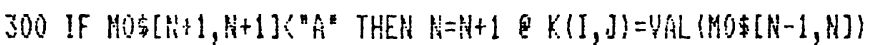

$310 k(1,1)=f(1,3)+10$

320 REXT H.

330 NEXT J

340 65S16! 1 TO "DATEIO"

350 FRINT $1 ; E, H$

360 FOA $1=1$ TO $E$

370 FOF $J=1 \quad 50 \mathrm{M}$

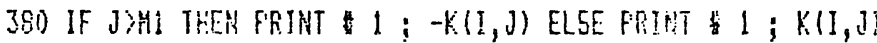

390 REXT $]$

400 YEXYT 1

410 DISP AS

420 CALL "DTÜFAH"

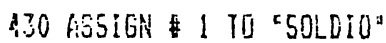

440 READ 1 ; $S$

450 IF $5=0$ THEW UISF "NO EXISTE SCLUCION - E EQTO 640 
460 IF $S>1$ THEN 650

470 FOR $J=1$ TO $\mathrm{H}$

430 KEAD $1 ; X(J)$

490 AEXT $J$

500 Fit $="$ "

510 FUR $J=1$ TO 4 \& DISF $X(1) ;$ \& NEXT $\mathrm{J}$

520 DISP

530 FOS $J=1$ TO $\mathrm{H}$

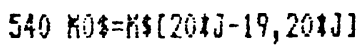

$5501 .=-05(\mu 0+, ")$

$560 \mathrm{Y0}:=M 0 \leqslant[1, L-1]$

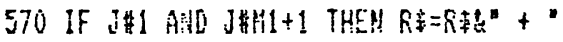

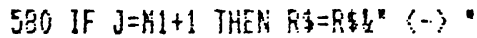

590 IF $X(J) \div 1$ THEN F $\$=R \& S T$ Th $\$(X(J))$

$500 \quad F \$=F+40 \%$

b10 HEXT J

$62 !$ DISF 51 STO

630 STOF

640 END

350 FOR $I=1$ TO 5

660 FOR $J=1$ TO $\mathrm{H}$

670 REFD $+1 ; K(1, J)$

G8O NEXT J

690 NEXT I

700 DISF "EXISTEH INFIHITAS SOLUCIOHES"

$710 \mathrm{~L}=0$

720 FOR $\mathrm{J}=1$ TO H

$730 L 1=0$

740 FOR $I=1$ TOS

750 If $(1, J)$ ? THEN $L 1=L 1+1$ \& $L 2=1$

760 MEXT 1

770 15 $L I=1$ THEH $L J=S G$ G $(K(L 2, J))$ \& $L=F M M(L, L 3+K(L 2, J)$ ELSE GOTO 790

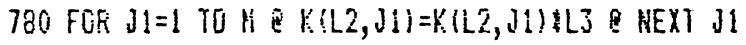

790 HEXT J

800 FOn $P O=1$ TO L

810 FOR $I=1$ TO $S$ E $P(I)=0$ \& NEXT I

$820 I=1 \& F(S)=P(A-1$

$830 P(I)=P(1)+1$ \& IF $P(1)<=F O$ THEN 250

$840 \mathrm{~F}(\mathrm{I})=0 \quad 1=1-1$ \& IF I THEN 830 ELSE 950

850 IF ISS THEH $I=1+1$ 60T0 830

860 FOR $J=1$ TO $\mathrm{H}$

$870 Y(J)=0$

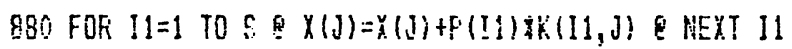

870 if $x(J) \leq=0$ on hod $(y(d)$, L) there 830

900 NEXT J

$910 \mathrm{LI}=0$

526 FOF $J=1$ TO $Y$ \& LI=FAM $(L 1, Y(J))$ HEXT J

930 FOR $J=1$ TO $H \in X(3)=x(3) / L \perp$ HEXT J

940 60TD 500

50 REXT PO

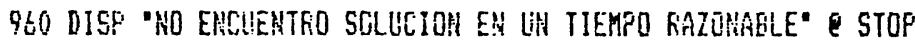

970 END

980 DEF FHH $(A, B)$

$990 A 1=A B S(A) \cup B 1=A B S(B)$

1000 IF $A 1<B 1$ THEN $B 1=A E S(A)$ \& $A 1=F_{B} B S(B)$

1010 IF $B 1=0$ THEN FIHA $=A 1$ E GOTO 1050

$\$ 020 \mathrm{Cl}=\mathrm{BOD}(\hat{\mathrm{A}} 1, \mathrm{B1})$

1030 IF CI THEN Al=E! BII=CI G0IO 1020

1040 FHYY $=6 !$

1050 END DEF 